were moved freely by oil. On the 13 th he was very much better. On the 20 th he occasionally complained of pain still on the right side. On the $23 \mathrm{rd}$ a distinct swelling had developed in the right lumbar region. It was fairly large and fluctuant, and slightly moveable. There was no redness of the skin, but some tenderness was present.

An operation was performed by me on May 26th. The swelling was punctured and was found to contain clear fluid which resembled urine. An incision was then made in the lumbar region and the muscular wall was divided down to the kidney. This was defined, and it was noted that there had been a laceration of the kidney, which had cicatrised. A tube was inserted, and the wound was stitched.

On May 30 th the patient was very well. There was a considerable discharge of clear fluid from the wound. On June 7th the tube was left out. On the 14th there was no discharge of urine from the wound. On the 20th the boy was dismissed well to the convalescent home, the urine being quite normal.

Remarks.-These two cases resemble each other in the following points. (1) Both patients fell on the loin ; (2) the injury in both cases was direct-one a fall on the edge of a stair, the other on the edge of a pail ; $(3)$ in both blood was present in the urine immediately after the accident; (4) in both the ruptured kidney could be felt by palpation, in each case extending round the abdomen towards the umbilicus and tender to touch; and (5) in both the swelling was clearly behind the peritoneum.

Glasgow.

\section{ON A NEW TEST FOR SUGAR.}

BY H. J. H. FENTON, So.D., F.R.S. LECTURER ON CHEMISTRY, UNIVERSITY OF CAMBRIDGE,

AT a meeting of the Cambridge Philosophical Society on Nov. 26th, 1906, I described a new method for the detection of sugars. The test is one of great delicacy, and though its application requires some manipulative skill it possesses certain advantages over many of those which have hitherto been employed. It appeared at that time that the test was only applicable to carbohydrates in the solid form, but on further investigation I have found that with a slight modification it may be used for the detection of sugars in aqueous solution. Even in this modified form the reaction is sufficiently delicate to detect 0.2 per cent. or less of sugar in urine or in similar liquids.

The reaction depends upon the fact that all carbohydrates of the hexose, or polyhexose, type (such as dextrose, lævulose, cane sugar, milk sugar, or maltose) yield a certain amount of bromo-methyl-furfural, $\mathrm{CH}_{2} \mathrm{Br}$ $\mathrm{C}_{4} \mathrm{H}_{2} \mathrm{O}-\mathrm{CHO}$, when acted upon by hydrobromic acid under appropriate conditions; ${ }^{1}$ and further, that the latter substance reacts with malonic ester in presence of alkalis, giving rise to a product the solutions of which exhibit a powerful blue fluorescence.

The conditions most favourable for the action of hydrobromic acid are obtained when phosphorus tribromide is dissolved in some inert solvent, such as toluene, and the solution is heated with the carbohydrate in presence of water ; excess of water, however, must be avoided.

For the examination of urine the test is most conveniently applied in the following way. Pour a small quantity (four or five cubic centimetres) of the liquid on to an excess of solid anhydrous calcium chloride so as to form a semi-solid, or pasty, mass. Add to this ten cubic centimetres of toluene containing two or three drops of phosphorus tribromide and then carefully boil the mixture for a few minutes, bearing in mind the inflammable nature of toluene. Pour off the toluene solution and, after cooling, add to it about one cubic centimetre of malonic ester and a little alcohol. On neutralising the mixture by adding alcoholic potash, drop by drop, a characteristic pink colour will usually be observed. The mixture is now considerably diluted with alcohol and a few drops of water when, if sugar was originally present, the solution will exhibit a beautiful blue fluorescence.

The reaction appears to be a specific one for carbohydrates which contain six or more atoms of carbon in the molecule and may therefore be used to distinguish hexoses from pentoses or other lower sugars.

Cambridge.

1 Transactions of the Chemical Society, 1898, p. 554.
OBSERVATIONS ON THE INDUCTION OF AUTO-VACCINATION IN TUBERCULOSIS AND OTHER CHRONIC GLANDULAR INFECTIONS BY THE X RAYS

(AS REVEALED BY THE OPSONIC CHART OF THE FORMER).

By H. D. McCULLOCH, M.B., C.M. GLAsG.,

LATE CHIEF MEDICAL OFFICER OF THE HYDERABAD STATE RAILWAY HOSPITALS, SECUNDERABAD.

Mr attention was drawn last August to Dr. Peter Paterson's article which appeared in THE LANCET of August 25th, 1906, pp. 493, 494, entitled "Some Observations on the Effect of Sterile Caseous Matter in the Treatment of Tuberculous Disease." Dr. Paterson succeeded in jsolating an immunising agent from the chronically diseased tuberculous lymphatic glands, with which he vaccinated six patients suffering from extensive tuberculous disease these cases were such that the application of the new tuberculin, or T.R. of Koch, even in minimal doses was obviously contra-indicated, owing to the unavoidable successive auto-inoculations in generalised tuberculous lesions His results were gratifying without exception. They show three things: (1) that positive phases of opsonic indices were easily obtained without negative phases (though here the possibility of an elision of the negative phase or of its duration being so brief as to escape observation must be borne in mind) ; (2) that each rise in the positive phase was accompanied by minute elevation of temperature, this serving as a sufficient and reliable guide to dosage; and finally (3) that the normal index, which was soon reached, was easily maintained within normal limits without great or rapid variations. These three facts make it highly probable that this immunising agent or locally elaborated vacoine is an ideal one, and one which it would not be possible to prepare in the laboratory any more than it would be possible to prepare the vaccine for variola artificially

In the light of such knowledge it would seem that there is a very marked and important difference between the opsonic response evoked by vaccination with this product, which is elaborated locally in vivo, and that evoked by new tuberculin, or T.R. of Koch, which, with all deference to Professor Koch, is not a metabolic defensive product, and therefore not a true vaccine, while that of Fdward Jenner has so far stood alone as being a true vaccine, being obtained vicariously by virtue of the action of the very minute lymphatic glands in the vicinity of the inoculated area prior to the processes of complete encapsulation. It is conceivable that the ideal vaccine to the tubercle bacillus will be one elaborated in vivo, and obtained from the sterile accretion produced by the phagocyted tubercle bacilli found in the glandular focus of an otherwise untainted animal which has been inoculated by a certain otherwise sterile strain of the human tubercle bacillus. The definition of a true vaccine would therefore be that it is a metabolic lymphatic glandular product, elaborated in the organism in consequence of a completed final defensive reaction. This would follow upon any organic infectious inoculation, produced either accidentally or intentionally.

From what follows we may reasonably infer that in tuberculosis varying degrees of infection occur accidentally, invariably producing both immediate and remote effects among those whose conditions of environment (as in the case of Europeans), constantly expose them to this infection, some more and some less. Where these conditions of environment are lacking infections of this disease do not occur, as in the case of negroes and aborigines in their native haunts. On the occurrence of slight degrees of infection the preliminary defensive mechanisms suffice to destroy it, without necessarily effecting any appreciable change in the individual's health. The local and systemic effects may be so slight as to escape observation; but at the same time, by virtut of a definite opsonic response having been evoked, a slight degree of resistance is conferred on the individual. Periodic repetitions of such degrees of infection suffice thus to produce an acquired partial immunity-as for example, in Europeans, which infection is not the case with negroes and aborigines, since the disease does not exist amongst them in their native haunts. Thus it follows that the conditions of environment conducing to the acquirement of a natural immunity also conduce to the acquirement of the disease itself. The severity of the disease depends on 
the extent of immunity obtained prior to infection and upon the degree of infection when it occurs. I might point out incidentalls that the definite characteristics of both the degree of resistance and that of the opsonic defect would be transmitted to succeeding generations according to the Mendelian law of transmission.

In the light of present clinical knowledge I shall now briefly sketch the more important defensive mechanisms which are brought into play on the occurrence of an unmixed tuberculous infection of moderate severity in the case of an otherwise healthy (?) adult European. The preliminary defensive mechanisms having been overcome, we have to con sider, then. the extent and virulence of the infection on the one hand, and the vital resistance of the individual on the other, whether this be acquired or inherited. The site of infection may be on the mucous membrane of the floor of the buccal cavity or in the region of the tonsils, where the accidental presence of other bacilli among the normal flora of the buccal cavity influences the tubercle bacilli favourably or unfavourably, according as the accidental bacilli are certain pneumococci or streptococci which are congenial to the tubercle bacillus, or others which are not. It is well known in regard to this point that the tetanus bacillus, which is normally anaernbic, will nevertheless proliferate easily under aerobic conditions on a culture medium in company with several common saprophptic organisms, such as the ordinary bacteria of decomposition found in a foul lacerated wound. After adapting themselves to their altered environments in such localities and surviving a definte period of incubation the tubercle bacilli tend to undermine the adjacent structures and to move from their extra-corporeal position to other positions which are intra-corporeal. On the one hand, the opsonins present begin to be expended and a further response is evoked; on the other hand, the mechanisms of phagocytosis and segregation are now simaltaneously brought into play by virtue of a leucocytosis in which the lymphatic system is mainly concerned. There are evidences of a hyperæmia at the site of infection, elevation of the normal bodily temperature, and $a$ fall and rise of the opsonic index, according to the fundamental laws laid down by Sir A. E. Wright. A migration of at least two types of leucocytes takes plare in the vicinity of the infection-one of these being concerned with the formation of neopla-tic encapsulating tissue for the purpose of segregation and the other type with the carrying on of phagocytosis. With reference to this action it is conceivable that the phagooytes deal with the more advanced and scattered bacilli, especially those that are opsonised, while the other type surround those that are massed together so as to form the tubercles or localised foci and keep them inclosed pending the development of further opsonins and incitor elements.

The expenditure and generation of opsonins depends (1) upon the regulation and degree of mobility of the invading forces; (2) npon the rate and efficiency of the segregations; and (3) upon their location, whether they are in or near to highly vascular tissues (where they are more accessible to the leucocytes) or in remote, less vascularised tissnes. The tissues of the adult, being more organised and dense, offer a greater obstacle to the migration of leucocytes in unwonted directions, but the less organised tender tissues of the infant are more permeable. Phagocy tosis having been completely effected after opsonisation, and encapiulation established, the acute stage of the malady is tided over and the more subtle, chronic stage of convalescence sets in Where the encapsulating tissues contain opsonised bacilli only these bacilli probably undergo degeneration and die and if the encapsulating tissues be not too dense to permit of the slow liberation and diffusion of their toxins a stimulus to incitor elements is produced and the phagocytes are incited to penetrate those foci from which they do not emerge again, but the contents of which they neutralise, so forming pus. But there may also be some active foci, the bacilli in which have escaped opsoni-ation previously to encapsulation; these in due course proliferate and sub. sequently emerge on molerate bolily exertion, causing autoinoculations, probably before the system has had time to recuperate from its losses and an exacerbation of the malady occurs. To meet this contingency, however (if the sugg:stions implied by Dr Paterson be sound), pari passu with the phenomena described above, an elaboration of a tuberculo-tropic and antimicrobic vaccine has taken place in the lymphatic glands corresponding to the sites of the original infection. At this stage of the malady these glands are densely, and therefore more or less imperviously, encapsulated in the neoplastic tissues. These glands having performed this important function now cease to act and become degenerated and casseated. The afferent and efferent vessels are blocked within and constricted without. The egress of this substance into the circulation is thus barred when it is most needed and recovery depends on its regulated or accidental admission into the circulation. After the surgical removal of certain tuberculous glands we may find in the unintentional vaccination thus effected an explanation for the occasional disappearance of tuberculous symptoms. This action is similar to the case of certain intra abdominal suspiciously cancerous tumours with glandular involvement, where, after opening the peritoneal cavity. the tumour mass for certain reasons has been left without further operative procedure and in a remarkable manner has been known to disappear subsequently. I merely offer these suggestions on the grounds of the bigh potency of an al most infinitesimal quantity of such vaccine, where present in the lymphatic system, and the possibility of its accidental admission into the circulation.

It is the purpose of tbis paper to attempt to demonstrate the theory that the mechanism of the therapeutics of $x$ rays rests upon the induction of an auto-vaccination, subsequent to the resolvent action of the $x$ rays on the rudimentary neoplastic encapsulating tissues abont the tuberculous glands, thus rendering the vaccine accessible to the bloodstream. It will also be shown how its effects may be observed by minute temperature reactions, accompanied by similar advances in the opsonic index without negative phases, tested by the periodic estimation of the opsonic index to tubercle bacillus. As a preliminary it will be advisable to give some general account of the effects of $x$ rays upon normal and abnormal tissues.

The $x$ rays are known to have a special selective affinity for morbid tissues, independently of their position or of any inflammatory reaction. This selective action on morbid tissue may proceed without any signs of irritation or any apparent modification of the skin. The dose necessary for the production of an alopecia will naturally be less than that required for the destruction of the granulation tissue of an epithelioma. In the use of other therapeutic agents we are often obliged to vary the dose. A given drug may have a tonic action in a small dose, an emetic action in a moderate dose, and a poisonous action in a large dose. The same may be said of the $x$ rays. Take, for example, their action on the scalp. In small fractional doses they have a stimulating effect on the hair, produring an action similar to that of a rubefacient lotion; whereas in large doses they cause epilation, and in still larger doses vesiculation, ulceration, and sloughing. Again, it is well known that certain drugs have a tendency to accumulate in the system and that in this way minute doses frequently repeated may produce serious results. To some extent this also is true of the $x$ rays when frequently repeated doses are applied over the same spot, but not when these doses are distributed over the body, seeing that the locally absorbed ions are not influenced by the general circulation. ${ }^{1}$

Many authorities both in this country and abroad have studied, post mortem, the microscopic appearances of morbid tissues that have during life been subjected therapeutically to the action of $x$ rays, and they have sought to establish the mechanism of its curative effects.

In regard to the therapeusis of $x$ rays Dr. H. Lewis Jones ${ }^{2}$ says, "The gradual onset (of radio-dermatitis) after a latent period, suggests that the lesion ( $x$-ray dermatitis) is a trophoneurotic one due to the damage sustained by the superficial nerves of the part ;" and again later he says, "It will be noticed that nothing is here given on the appearances presented by cutaneous nerves, and this is probably an omission of importance."

Schwartz, working in Holzknecht's laboratory, has further pointed out that the $x$ rays decompose the lecithin in the neurolemma of the nerve filaments, producing methylamin. The inference would therefore be that primarily the nerve filaments are acted upon, producing a paresis of the capillaries, followed by a passive hyperæmia, and that secondarily an analgesia, and finally a necrosis, follow, with the usual line of demarcation. ascording to the quantity and the quality of the $x$ rays applied. The subsequent processes of repair, depending on the rate of regeneration of the trophic nerve filaments, are naturally very slow and tedious. 
Dr. J. Belot ${ }^{3}$ states in regard to lupus after irradiation: It was at this time that my attention was drawn to Dr. "The following is apparently the mechanism of cure by radiotherapy : the hyperæmia set up by the $x$ rays provokes an abundant migration of leucocytes from the vessels. As may be seen after a tuberculin (sio) injection, the leucocytic infiltration commences at the periphery of the tubercular focus and penetrates by narrow tracks into the substance of the lupus nodules, these leucocytes being ultimately transformed into fusiform cells and fibrous connective tissues. The presence of these fusiform cells bears witness to the active proliferation of connective tissue. The lupus cells degenerate by vacuolisation; the nuclei lose their affinity for stains, necrose, and become absorbed, the diseased tissue being replaced by cicatricial growth. As Schwartz has shown, all idea of bactericidal action falls to the ground The most important point in the case of lupus is the localisation of the inflammatory reaction and its concentration exactly at the diseased point, in consequence of the degeneration of the lupus nodules themselves. Both Schwartz and Neisser pointed out that there is in this respect a resemblance between the action of $x$ rays and that of tuberculin, which also sets up inflammatory phenomena that are concentrated on the tuberculous nodules."

It is well known that all neoplastic tissue is a rudimentary replica, almost in every detail, of the tissue affected, and that such tissues are highly susceptible to the influence of $x$ rays and of the less penetrating cathode rays. These stop short of damage to the normal and more organised tissuts, Paterson's article. Shortly afterwards another of these case of enlarged tuberculous glands came up for treatment and 1 was enabled by the kindness of Dr. W. E. Facey and Mr. A. B. Batley of Christchurch to have the patient's opsonic index estimated previously to, and during, a series of irradiations by the $x$ rays.

The patient was a single woman, aged 27 years, an only daughter, both of whose parents were living and well. Her childhood had been spent in London where she bad acquired continuous throat trouble and inflammation of both tonsils, which had been partially removed at the age of 11 years. She had suffered much from anæmia as a girl and also from bronchitis and loss of appetite. The patient first came under my medical observation in April, 1906, suffering from inveterate constipation. In the month of August following she drew my attention to certain glandular swellings in the neck, more noticeable on the right than on the left side. She had been steadily losing weight during the previous six months Her record weight had been 9 stones 8 pounds, and she was now 8 stones 2 pounds; her height was 5 feet 4 inches. The body, not so well nourished, but not emaciated, gave the appearance of one in ordinary good health. The temperature had remained subnormal for some months, ranging from $96^{\circ}$ to $98^{\circ} \mathrm{F}$. The patient's habits were inactive and she was easily fatigued but there were no further syn ptors of constitutional disease traceable. The opsonic index to tubercle

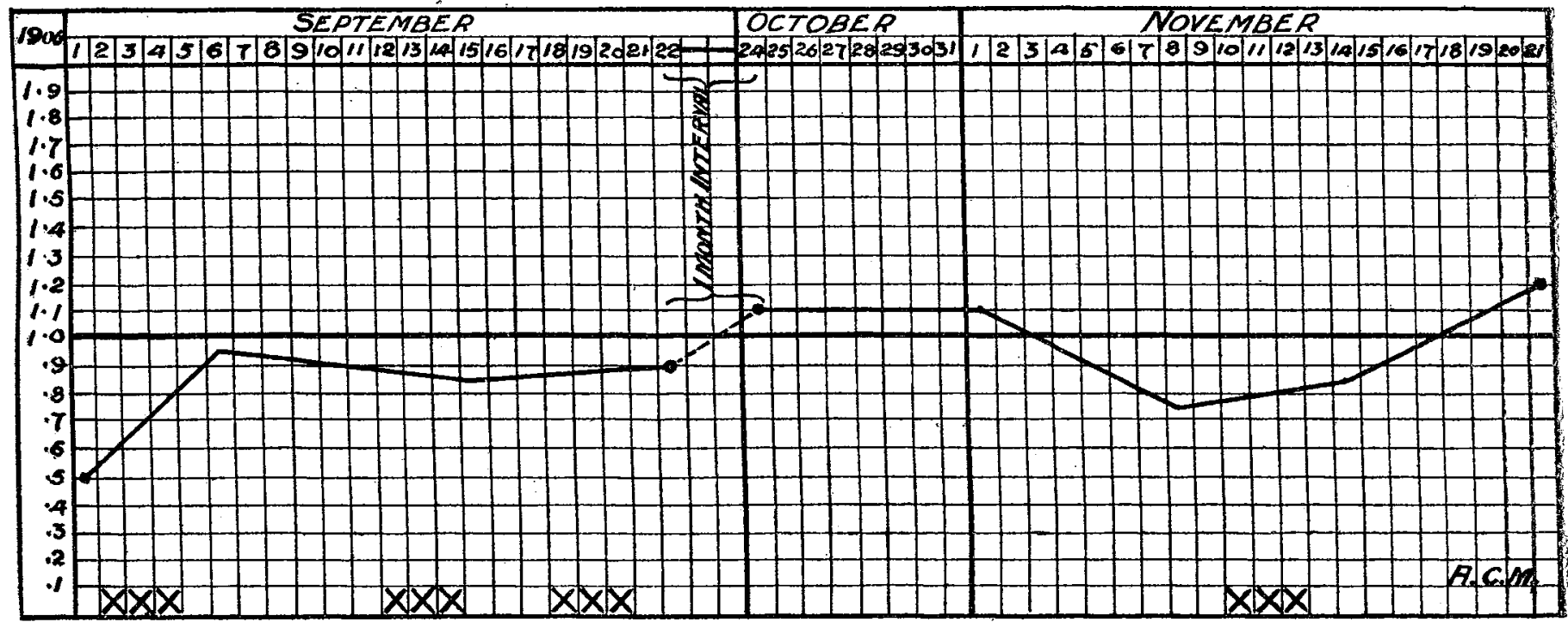

Chart showing the variations in the opsonic index.

when they are judiciously applied, and they restore metabolism where it has more or less or me to a standstill.

So much, then, for the therapeutic action of the $x$ rays on the normal and abnormal tissues. I now proceed to give cases occurring lately in my own practice upon which the theory of this paper is based. I wish to remark that during the past two years I have utilised these effects of the $x$ rays solely with a view to restoring the natural contour and to removing the disfigurement in females and males in such situations as the cervical, peribronchial and mediastinal regions. In the last case a mass of glands to the left of the sternum had produced an "aneurysmal" bulging of the third, fourth, and fifth sterno costal cartilages and marked cardiac displacement, \&c. The invariable results, I need hardly add, were a disappearance of the swellings after alternate soft swellings and shrinkings. Mild toxæmias in the intervals, as one would anticipate, without appreciable ri-es of temperature more marked, however, in the mediastinal case, did occur. No symptoms of re-infection were encountered, ant, from what has been above stated, this kogey need not be apprehended, except in early acute cases. On the other hand, these cases almost always without exception gained very appreciably in wejght and improved in their general health in a remarkable manner. I became curious to explain this fact and as none of their opsonic indices had been estimated it occurred to me that Dr. Paterson's suggestion might account for it.

3 Radiotherany, p. z07. bacilli on Sept. 1st was 0.5 , with brief irradiations on the 2nd 4th, and 6 th ; on the 6th it was 095 , with brief irradiations on the $12 \mathrm{th}, 13 \mathrm{th}$, and 14th ; on the $16 \mathrm{th}, 0.82$, with slight skin reaction and with brief irradiations on the $18 \mathrm{th}, 19 \mathrm{th}$, and 20th; on the 22nd, 0.90 (the patient had returned after a visit to London); on Oct. 26th, 1.11 ; on Nov. 1st, 1.09 (the patient throvghout the previous day had taken an active part in a bazaar) : on the $7 \mathrm{th}, 0.72$, with irradiations on the $10 \mathrm{th}, 11 \mathrm{th}, 12 \mathrm{th}$, and 13th; on the 14th. 0.82 ; and on the $22 \mathrm{nd}, 1.2$. During this period a gain in weight of $9 \frac{1}{4}$ pounds took place and a general improvement in health with the disappearance to external observation of the glands in the neck. The temperature had ranged between $97^{\circ}$ and $99^{\circ}$ earh irradiation being followed by a rise of from $05^{\circ}$ to $1^{\circ}$ in temperature. In the intervals of the irradiations the gla nos noftened, swelled, and shrank, with tenderness on press nie until they disappeared.

This case is not considered cured nor as yet completed, but the technique necessary for continuing to obtain the opsonic index in this, or in a number of cases, necessari] has its limitations, owing to the expense incurre $d$ in obtaining the opsonic index, a matter of about $£ 20$ in each case. This method can therefore only be employed in occasional cases where further proof of this important effect of treatment by the $x$ rays may be desired.

The limitation of the phases of the opsonic curre within. narrow and normal limits - the entire absence of negative phases, and finally, the minute range of the temperatinte 
reactions go far, in the opinion of the writer of this paper, to render his theory worthy of consideration.

The radio-therapeutic technique, with radiosometric chart, I propose submitting in due course to Medical Electrology and Radiology. in the October number of which journal a significant article from the pen of Dr. H. B. Wilkinson, physician in charge of the San Lazaro Hospital, Manila, appears in an abstract. under the title "The Treatment of Leprosy with the $X$ Rays," to which I would direct the attention of those interested in advanced therapeutics. Of this I will quote one sentence : " Another case was well after 14 treatments. The writer is inclined to believe that when a local lesion of leprosy is treated with the $x$ rays the organisms there localised are killed, and their bodies are absorbed into the system, thereby producing an immunity of the system against the living organisms."

Holzknecht says : "The cure of an infective process is not due to any bactericidal action of the $x$ rays, since the bactericidal dose is many times as great as the curative dose." This theory in regard to the mechanism of $x$ rays is quite compatible with the theory of the parasitic origin of cancer. "Skinner has given a detailed résumé of the hypotheses put forward to explain the cure of malignant tumours by the $x$ rays. ...... According to the third hypothesis cancer is a parasitic disease, and the $x$ rays destroy or hinder the development of the parasites. It may be, however, that the $x$ rays are not destructive to the parasite itself, bat in some way modify the tissues so as to prevent its development." $t$

In this reference, Dr. G. H. A. Clowes of the New York State Cancer Laboratory, in his article entitled "A Study of the Influence exerted by a Variety of Physical and Chemical Forces on the Virulence of Carcinoma in Mice" $\bar{s}$ states: "That immune forces are produced in the serum in gradually increasing quantities as the tumour progresses is indicated by the tendency frequently exhibited by tumours to remain stationary, after fairly rapid development, and then subsequently either to grow rapidly or retrogress. In this connexion it must be noted that $x$ rays produce a much more marked effect at this stage, at which apparently the tumour (?) has quite overcome the natural immunity of the mouse, and is developing rapidly."

Dr. Belot thus sums up: "In conclusion, we may say that the $x$ rays exercise a special action on certain cancerous cells, causing their degeneration and absorption, but that the mechanism of the action is still unknown."

I am tempted by the obvious therapeutic parallelism in the recently reputed medicinal agents in cancer to enter into their probable therapeutic values as recorded in the experience of others. I refer to papain, trypsin, and amylopsin, but I will only add that these enzymes, when injected in emulsion into the encapsulating tissues where metabolism has come to a standstill, bring about active resolution (if veins are avoided) by virtue of their proteolytic and diastatic properties on those tissues, when the lesions are accessible and not too deeply situated. They may thus liberate the vaccine when present in the lymphatic glands with the desired result if they do not influence it unfavourably by admixture before neutralisation.

Clinical experience is greatly in advance of the theories hitherto put forward, and I am not in a position at present to make manifest the above theory "by columns of figures and percentages of calculations of cures." I merely venture to submit these observations in the hope that if confirmed by those who have the necessary facilities for working in this department of therapeutics they will afford a solid basis for the scientific employment of radiotherapy, as well as for the proper classification of those conditions in which its use is indicated, and a most important field of medication will thus be opened up among those chronic ailments which occur after middle life and in which ordinary therapeutic measures have so far failed.

Bournemouth.

Belot's Radiotherapy. 5 Brit. Med. Jour., Dec. 1st, 1906, p. 1553.

British Electro-Therapeutic Society.-The annual general meeting of this society will be held at 11, Chandos-street, Carendish-square, W., to-day (Friday, Jan. 25th) at 830 P.M., when the election of officers of the society for 1907 will take place. Mr. J. Hall-Edwards, the retiring president, will deliver an address.

\section{TRACHEOTOMY IN SLIGHT RESPIRATORY OBSTRUCTION ASSOCIATED WITH FEBRILE TOX EMIA.}

BY A. O. BISSON, M.R.C.S. ENG., L.R.C.P. LOND., JUNIOR ASSISTANT MEDICAI OFFICER, PLAISTOW FEVER HOSPITAL.

IN fever practice there is a type of case occasionally encountered in which toxæmia is accompanied by very slight -apparently inconsiderable-obstruction of respiration. Cases of this kind are perhaps commonest in septic scarlet fever with swelling in the faucial region and the nasopharynx, possibly with some degree of septic laryngitis. A somewhat similar condition, however, is now and then seen in small-pox; one case of this type occurred at Dagenham Hospital during the last epidemic in London. The laryngitis caused a slight degree of obstruction which, owing to the enfeebled state of the patient, soon began to tell on his cardiac condition. Tracheotomy was performed; it was followed by a remarkable improvement and the patient recovered. There is also a septic type of diphtheria defined by Monti in which the larynx is but little affected; there is slight obstruction but it is not progressive and the patient apparently goes downhill as a result of the toxæmia alone. Some years ago, in a case at Plaistow Hospital, the question arose whether the patient's steady loss of ground might not be in part the result of very slight respiratory obstruction - strain on the respiratory muscles and deficiency of oxygen. The result of tracheotomy was a striking im. provement in the condition of the patient with ultimate recovery where it seemed hardly possible. In consequence of this result it was laid down as a principle that no patient with slight respiratory obstruction should be allowed to die without tracheotomy having been resorted to. From time to time such cases have occurred at Plaistow Hospital ; some of them have been under my care, and it may be of service in calling attention to a procedure so far not emphasised in medical literature. Its importance cannot be measured by the frequency of the cases in which it applies, because when such cases do occur they are of a grave nature, and the operation, as $I$ hope to show, is sometimes life-saving.

\section{INDIOATIONS FOR OPERATION.}

The indications for tracheotomy in the type of case under consideration are :-

1. Difficult breathing.-Owing to the intense congestion of the fauces and the profuse muco-purulent discharge from the naso-pharynx insufficient air reaches the lungs. The patient may breathe with the mouth open and the lower jaw often drops during each inspiration. The breathing is noisy owing to gurgling of the accumulated discharge in the pharynx, stridor being seldom present. The respirations are usually increased in frequency and the alæ nasi are brought into action; this increase in frequency is not marked and as in definite laryngeal obstruction the respirations may be slower than one might expect from the height of the temperature and the rapidity of the pulse. Apart from the swelling in the pharyngeal region there is not uncommonly marked lymphadenitis on both sides of the neck; this is often accompanied by cedema and even in some cases by an acute inflammation of the subcutaneous tissues of the neck; the result may then be pressure on the trachea, an exceptional indication for operation. It is necessary to lay stress on the fact that the obstruction is usually extremely slight and that a priori there might seem little hope of material benefit from operation. It would appear, however, that it is its duration, possibly extending to several days, which tells on the general condition and, especially, puts a strain on the heart.

2. Restlessness. - This is partly due to the difficult breathing; the patient is often delirious or semi-conscious and does not seem to realise that his respiration is laboured, yet he may appear obviously distressed. He often tosses about uneasily, pushes away the bed-clothes, and sometimes tries to sit ap. He may be unable to sleep, sedatives having very little effect. The condition is, of course, one that must tell on the already toxin-weakened-perhaps partly asphyxiated - heart. The distress often increases considerably when the patient attempts to drink or during applications to the throat and nose. At such times there may be coughing, return of fluid through the nose, cyanosis, exhaustion so great that treatment has to be stopped, feeding by the mouth discontinued, and rectal feeding resorted to. 